PROCEEDINGS OF THE

AMERICAN MATHEMATICAL SOCIETY

Volume 132, Number 8 , Pages $2303-2314$

S 0002-9939(04)07331-9

Article electronically published on February 26, 2004

\title{
A WOLD-TYPE DECOMPOSITION FOR COMMUTING ISOMETRIC PAIRS
}

\author{
DAN POPOVICI
}

(Communicated by Joseph A. Ball)

\begin{abstract}
We obtain a Wold-type decomposition theorem for an arbitrary pair of commuting isometries $V$ on a Hilbert space. More precisely, $V$ can be uniquely decomposed into the orthogonal sum between a bi-unitary, a shiftunitary, a unitary-shift and a weak bi-shift part, that is, a part $S=\left(S_{1}, S_{2}\right)$ that can be characterized by the condition that $S_{1} S_{2},\left.S_{1}\right|_{\bigcap_{n \geq 0}}$ ker $S_{2}^{*} S_{1}^{n}$ and $\left.S_{2}\right|_{\cap_{n \geq 0}} \operatorname{ker} S_{1}^{*} S_{2}^{n}$ are shifts. Moreover, $S$ contains bi-shift and modified bi-shift maximal parts.
\end{abstract}

\section{Preliminaries}

Wold introduced, in a probabilistic language, a remarkable decomposition for stationary stochastic processes ([Wo $)$. Separating the deterministic part by the part corrupted by noises, this decomposition becomes the cornerstone of prediction theory for this kind of process. Other famous mathematicians, including von Neumann, Kolmogorov, and Halmos, formulated Wold's result for isometric operators on Hilbert spaces: the study of such operators is reduced to the study of unitary operators (which are well understood: we have the associated spectral theory and functional calculus) and of unilateral shifts (which have a very simple geometrical structure).

A (unilateral) shift is an operator $S$ on a Hilbert space $\mathcal{H}$ unitarily equivalent to multiplication by the independent variable $z$ on a certain Hardy space on the torus. More precisely, there exist a Hilbert space $\mathcal{W}$ and a unitary operator $U: \mathcal{H} \rightarrow$ $H^{2}(\mathbb{T}) \otimes \mathcal{W}$ such that $S=U^{*}\left(T_{z} \otimes I\right) U$ (the symbol " $\otimes$ " denotes the Hilbertian tensor product). The following characterization illustrates the shift's geometrical structure: an isometry $S$ on $\mathcal{H}$ is a shift if and only if there exists a wandering subspace $\mathcal{W}$ (i.e., $S^{n} \mathcal{W} \perp S^{m} \mathcal{W}, n, m \geq 0, n \neq m$ ) that generates $\mathcal{H}$, that is, $\mathcal{H}=\bigoplus_{n \geq 0} S^{n} \mathcal{W} . \mathcal{W}$ is unique $\left(\mathcal{W}=\operatorname{ker} S^{*}\right)$ and is said to be the defect of $S$.

An operator $V$ on $\mathcal{H}$ is said to be reduced by a (closed) subspace $\mathcal{H}_{0} \subset \mathcal{H}$ if $\mathcal{H}_{0}$ is invariant under both $V$ and $V^{*}$, i.e., $V \mathcal{H}_{0} \subset \mathcal{H}_{0}$ and $V^{*} \mathcal{H}_{0} \subset \mathcal{H}_{0}$.

Received by the editors September 13, 2002 and, in revised form, April 21, 2003.

2000 Mathematics Subject Classification. Primary 47A13, 47A45.

Key words and phrases. Wold-type decomposition, (dual) bi-isometry, (weak, modified) bishift, unitary extension.

This work was supported by the EEC Research Training Network: "Analysis and Operators", contract no. HPRN-CT-2000-00116. 
Wold's Theorem (see [NF1, Chapter 1). To any isometry $V$ on $\mathcal{H}$ there corresponds a unique orthogonal decomposition of the form

$$
\mathcal{H}=\mathcal{H}_{u} \oplus \mathcal{H}_{s}
$$

such that $V$ is reduced by $\mathcal{H}_{u}$ to a unitary operator and by $\mathcal{H}_{s}$ to a shift. More exactly, $\mathcal{H}_{u}=\bigcap_{n \geq 0} V^{n} \mathcal{H}$ and $\mathcal{H}_{s}=\bigoplus_{n \geq 0} V^{n} \mathcal{W}$, with $\mathcal{W}=\operatorname{ker} V^{*}$.

We want to underline the importance of this decomposition in dilation theory, invariant subspace theory, prediction theory, and operator interpolation problems. It is expected that such a result for several commuting isometries would provide a larger class of applications. Some steps forward, which motivate our present work, have already been made: certain multi-dimensional Wold-type decompositions have been applied in index theory for $\mathbf{C}^{*}$-algebras $([\mathrm{BCL}])$ or invariant subspace theory $(\underline{\mathrm{KO}})$.

One of the first attempts to extend the Wold theorem was made by Słociński St], who provided conditions on a commuting isometric pair in order to obtain a fourfold Wold-type decomposition of the form unitary-unitary, unitary-shift, shift-unitary and shift-shift. In particular, his result applies for double commuting isometries, that is, isometric pairs $\left(V_{1}, V_{2}\right)$ such that $V_{1}$ commutes not only with $V_{2}$ but also with the adjoint of $V_{2}\left(V_{1} \operatorname{ker} V_{2}^{*} \subset \operatorname{ker} V_{2}^{*}\right.$ or, equivalently, $\left.V_{2} \operatorname{ker} V_{1}^{*} \subset \operatorname{ker} V_{1}^{*}\right)$.

A natural extension for the unilateral shift is the notion of bi-shift, namely a pair $S=\left(S_{1}, S_{2}\right)$ on a Hilbert space $\mathcal{H}$ unitarily equivalent to the pair of multiplications by coordinate functions $z_{1}$ and $z_{2}$ on a certain Hardy space on the bitorus. More precisely, there exist a Hilbert space $\mathcal{W}$ and a unitary operator $U: \mathcal{H} \rightarrow H^{2}\left(\mathbb{T}^{2}\right) \otimes \mathcal{W}$ such that $S_{i}=U^{*}\left(T_{z_{i}} \otimes I\right) U, i=1,2$. The following characterization illustrates the geometrical structure of a bi-shift [St]: a commuting isometric pair $S=\left(S_{1}, S_{2}\right)$ on $\mathcal{H}$ is a bi-shift if and only if $S$ is a doubly commuting shift pair if and only if there exists a wandering subspace $\mathcal{W}$ (i.e., $S^{p} \mathcal{W} \perp S^{q} \mathcal{W}, p, q \in \mathbb{Z}_{+}^{2}, p \neq q$ ) that generates $\mathcal{H}$, that is, $\mathcal{H}=\bigoplus_{p \in \mathbb{Z}_{+}^{2}} S^{p} \mathcal{W}$ (if $p=\left(p_{1}, p_{2}\right) \in \mathbb{Z}^{2}$ and $T=\left(T_{1}, T_{2}\right) \in \mathcal{L}(\mathcal{H})^{2}$ is a commuting pair we use the notation $T^{p}$ for: $T_{1}^{p_{1}} T_{2}^{p_{2}}$ if $p_{1}, p_{2} \geq 0 ; T_{1}^{*\left|p_{1}\right|} T_{2}^{p_{2}}$ if $p_{1}<0, p_{2} \geq 0 ; T_{2}^{*\left|p_{2}\right|} T_{1}^{p_{1}}$ if $p_{1} \geq 0, p_{2}<0$; or $T_{1}^{*\left|p_{1}\right|} T_{2}^{*\left|p_{2}\right|}$ if $\left.p_{1}, p_{2}<0\right)$. $\mathcal{W}$ is unique $\left(\mathcal{W}=\operatorname{ker} S_{1}^{*} \cap \operatorname{ker} S_{2}^{*}\right)$ and is said to be the defect of $S$.

We are now able to formulate

Słociński's Theorem ([St] $)$. To any double commuting isometric pair $V=\left(V_{1}, V_{2}\right)$ there corresponds a unique orthogonal decomposition of the form

$$
\mathcal{H}=\mathcal{H}_{u u} \oplus \mathcal{H}_{u s} \oplus \mathcal{H}_{s u} \oplus \mathcal{H}_{s s}
$$

such that $\mathcal{H}_{\alpha_{1} \alpha_{2}}$ reduces $V_{i}$ to a unitary operator if $\alpha_{i}=u$ and to a unilateral shift if $\alpha_{i}=s, i=1,2$. More exactly, $\mathcal{H}_{\text {uu }}=\bigcap_{m \geq 0} V_{1}^{m} \mathcal{H} \cap \bigcap_{n \geq 0} V_{2}^{n} \mathcal{H}, \mathcal{H}_{\text {us }}=$ $\bigoplus_{n \geq 0} V_{2}^{n}\left(\bigcap_{m \geq 0} V_{1}^{m} \mathcal{W}_{2}\right), \mathcal{H}_{s u}=\bigoplus_{m \geq 0} V_{1}^{m}\left(\bigcap_{n \geq 0} V_{2}^{n} \mathcal{W}_{1}\right)$ and $\mathcal{H}_{s s}=\bigoplus_{p \in \mathbb{Z}_{+}^{2}} V^{p} \mathcal{W}$, with $\mathcal{W}_{1}=\operatorname{ker} V_{1}^{*}, \mathcal{W}_{2}=\operatorname{ker} V_{2}^{*}$ and $\mathcal{W}=\mathcal{W}_{1} \cap \mathcal{W}_{2}$.

It is our aim in this paper to obtain such a Wold-type decomposition for an arbitrary commuting isometric pair, the bi-shift part being replaced by what we will call a weak bi-shift. This weak bi-shift contains, in particular, bi-shift and modified bi-shift maximal parts. 


\section{A generalized Wold-SŁOCińSKi DECOMPOSITION}

The first step in our construction is an important observation made by I. Suciu [Su, namely the existence of a maximal bi-unitary part for every commuting pair of isometries (in short, a bi-isometry). Some years later, Berger, Coburn and Lebow $\mathrm{BCL}$ stated that this decomposition between bi-unitary and completely non-unitary parts is exactly the Wold decomposition for the product isometry, a result that clarifies the structure of the spaces:

Proposition 2.1 ( $[\mathrm{Su}], \overline{\mathrm{BCL}}])$. Let $V=\left(V_{1}, V_{2}\right)$ be a bi-isometry on $\mathcal{H}$. There exists a maximal subspace $\mathcal{H}_{u u}$ of $\mathcal{H}$ reducing $V$ to a bi-unitary operator. Moreover, the orthogonal decomposition $\mathcal{H}=\mathcal{H}_{u u} \oplus \mathcal{H}_{u u}^{\perp}$ coincides with the classical Wold decomposition attached to the isometry $V_{1} V_{2}$; that is,

$$
\mathcal{H}_{u u}=\bigcap_{n \geq 0}\left(V_{1} V_{2}\right)^{n} \mathcal{H}
$$

$V$ is said to be completely non-unitary $\left(\right.$ cnu) if $\mathcal{H}_{u u}=\{0\}$.

In such a generality we identify a unitary-shift maximal subspace as in the Słociński decomposition theorem:

Proposition 2.2. There is a maximal subspace $\mathcal{H}_{\text {us }}$ of $\mathcal{H}$ reducing $V_{1}$ to a unitary operator and $V_{2}$ to a shift. More precisely,

$$
\mathcal{H}_{u s}=\bigoplus_{n \geq 0} V_{2}^{n}\left(\bigcap_{m \geq 0} V_{1}^{m}\left(\bigcap_{i \geq 0} \operatorname{ker} V_{2}^{*} V_{1}^{i}\right)\right)
$$

Proof. Since $\mathcal{W}_{2}:=\bigcap_{m \geq 0} V_{1}^{m}\left(\bigcap_{i \geq 0} \operatorname{ker} V_{2}^{*} V_{1}^{i}\right) \subset \operatorname{ker} V_{2}^{*}$ is wandering for $V_{2}$, we can define $\mathcal{H}_{u s}$ as the orthogonal sum in (2). Observe that, since the sequence $\left\{V_{1}^{m}\left(\bigcap_{i \geq 0} \operatorname{ker} V_{2}^{*} V_{1}^{i}\right)\right\}_{m \geq 0}$ is decreasing, its intersection remains unchanged if indexed by $m \geq 1$. Hence $V_{1} \mathcal{H}_{u s}=\mathcal{H}_{u s}$; that is, $\mathcal{H}_{u s}$ reduces $V_{1}$ to a unitary operator. Moreover, $V_{2} \mathcal{H}_{u s}=\mathcal{H}_{u s} \ominus \mathcal{W}_{2} \subset \mathcal{H}_{u s}$ and $V_{2}^{*} \mathcal{H}_{u s}=\mathcal{H}_{u s}$. Consequently, by the structure of $\mathcal{H}_{u s},\left.V_{2}\right|_{\mathcal{H}_{u s}}$ is a shift having $\mathcal{W}_{2}$ as the defect subspace.

Suppose that $\mathcal{H}_{0}$ is a subspace of $\mathcal{H}$ reducing $V_{1}$ to a unitary operator and $V_{2}$ to a shift. We shall prove that $\mathcal{H}_{0} \subset \mathcal{H}_{\text {us }}$. Since $\left.V\right|_{\mathcal{H}_{0}}$ is double commuting, we can use the geometrical structure in the Słociński decomposition:

$$
\mathcal{H}_{0}=\bigoplus_{n \geq 0} V_{2}^{n}\left(\bigcap_{m \geq 0} V_{1}^{m}\left(\operatorname{ker} V_{2}^{*} \cap \mathcal{H}_{0}\right)\right) .
$$

Observe that $V_{2}^{*} V_{1}^{i} x=V_{1}^{i} V_{2}^{*} x=0(i \geq 0)$, for any $x \in \operatorname{ker} V_{2}^{*} \cap \mathcal{H}_{0}$. Then $\mathcal{H}_{0} \subset \mathcal{H}_{u s}$, and the maximality is proved.

By symmetry we can also formulate

Proposition 2.3. There is a maximal subspace $\mathcal{H}_{\text {su }}$ of $\mathcal{H}$ reducing $V_{1}$ to a shift and $V_{2}$ to a unitary operator. More precisely,

$$
\mathcal{H}_{s u}=\bigoplus_{m \geq 0} V_{1}^{m}\left(\bigcap_{n \geq 0} V_{1}^{n}\left(\bigcap_{j \geq 0} \operatorname{ker} V_{1}^{*} V_{2}^{j}\right)\right) .
$$

Let $S=\left(S_{1}, S_{2}\right)$ be a bi-isometry on $\mathcal{H}$.

Remark 2.4. $S$ is bi-shift if and only if $S$ is double commuting and

$$
\left.S_{1}\right|_{\operatorname{ker} S_{2}^{*}},\left.S_{2}\right|_{\operatorname{ker} S_{1}^{*}} \text { and } S_{1} S_{2} \text { are shifts. }
$$


The direct inclusion follows by the observation that the restriction of a shift to any of its reducing subspaces remains a shift. For the converse, observe that, under the given hypothesis $\operatorname{ker} S_{2}^{*}=\bigoplus_{m>0} S_{1}^{m}\left(\operatorname{ker} S_{1}^{*} \cap \operatorname{ker} S_{2}^{*}\right)\left(\left.S_{1}\right|_{\operatorname{ker} S_{2}^{*}}\right.$ is a shift $)$ and $\operatorname{ker} S_{1}^{*}=\bigoplus_{n \geq 0} S_{2}^{n}\left(\operatorname{ker} S_{1}^{*} \cap \operatorname{ker} S_{2}^{*}\right)\left(\left.S_{2}\right|_{\operatorname{ker} S_{1}^{*}}\right.$ is a shift), we have

$$
\bigoplus_{p \in \mathbb{Z}_{+}^{2}} S^{p}\left(\operatorname{ker} S_{1}^{*} \cap \operatorname{ker} S_{2}^{*}\right)=\bigoplus_{m \geq 0} S_{1}^{m} \operatorname{ker} S_{1}^{*}=\bigoplus_{n \geq 0} S_{2}^{n} \operatorname{ker} S_{2}^{*}
$$

Also, by double commutativity, $\bigcap_{m>0} S_{1}^{m} \mathcal{H} \cap \bigcap_{n>0} S_{2}^{n} \mathcal{H}=\bigcap_{i>0}\left(S_{1} S_{2}\right)^{i} \mathcal{H}$. Hence $\bigcap_{m \geq 0} S_{1}^{m} \mathcal{H}=\bigcap_{n \geq 0} S_{2}^{n} \mathcal{H}=\bigcap_{i \geq 0}\left(\bar{S}_{1} S_{2}\right)^{i} \mathcal{H}=\{0\}$. Consequently, $\mathcal{H}_{s s}=\mathcal{H}$ (by Słociński's theorem) and $S$ is a bi-shift.

More generally, in the non-double commuting case we need to find a good replacement for the bi-shift operator as part of such a generalized Wold-Słociński-type decomposition.

Observe that $\bigcap_{i \geq 0} \operatorname{ker} S_{2}^{*} S_{1}^{i}$ (= $\operatorname{ker} S_{2}^{*}$ if $S$ is double commuting) is invariant under $S_{1}$, as well as $\bigcap_{j \geq 0} \operatorname{ker} S_{1}^{*} S_{2}^{j}$ (= $\operatorname{ker} S_{1}^{*}$ if $S$ is double commuting) under $S_{2}$. By the previous remark it is natural to introduce our bi-shift replacement by

Definition 2.5. $S$ is said to be a weak bi-shift if

$$
\left.S_{1}\right|_{\bigcap_{i \geq 0} \operatorname{ker} S_{2}^{*} S_{1}^{i}},\left.S_{2}\right|_{\bigcap_{j \geq 0} \operatorname{ker} S_{1}^{*} S_{2}^{j}} \text { and } S_{1} S_{2} \text { are shifts. }
$$

Recall that, for any contraction $T$ acting on a Hilbert space $\mathcal{H}$, there is a maximal subspace $\mathcal{H}_{u}$ of $\mathcal{H}$ reducing $T$ to a unitary operator ([La], [NF2] $)$. More exactly,

$$
\mathcal{H}_{u}=\left\{x \in \mathcal{H} \mid\left\|T^{n} x\right\|=\|x\|=\left\|T^{* n} x\right\|, n \geq 0\right\} .
$$

$T$ is said to be completely non-unitary $(c n u)$ if its corresponding space $\mathcal{H}_{u}=\{0\}$.

The definition above can be reformulated in order to better illustrate the weak bi-shift structure. We need the following:

Lemma 2.6. Let $S=\left(S_{1}, S_{2}\right)$ be a bi-isometry on $\mathcal{H}$. The following assertions are equivalent:

(i) $\left.S_{1}\right|_{\bigcap_{i}>0} \operatorname{ker} S_{2}^{*} S_{1}^{i}$ is a shift;

(ii) $S_{1}\left(I-S_{2} S_{2}^{*}\right)$ is cnu;

(iii) $\bigcap_{m \geq 0} S_{1}^{m} \mathcal{H} \cap \bigcap_{i \geq 0} \operatorname{ker} S_{2}^{*} S_{1}^{i}=\{0\}$.

By symmetry, the same kind of results are also true for $\left.S_{2}\right|_{\bigcap_{j \geq 0}} \operatorname{ker} S_{1}^{*} S_{2}^{j}$.

Proof. We prove first that $S_{1} \bigcap_{i>0} \operatorname{ker} S_{2}^{*} S_{1}^{i}=S_{1} \mathcal{H} \cap \bigcap_{i>0} \operatorname{ker} S_{2}^{*} S_{1}^{i}$. The direct inclusion is obvious. For the converse, consider $x \in S_{1} \mathcal{H} \cap \bigcap_{i>0} \operatorname{ker} S_{2}^{*} S_{1}^{i}$. Then $x=$ $S_{1} S_{1}^{*} x$ and $S_{2}^{*} S_{1}^{i} x=0, i \geq 0$. Consequently, $S_{2}^{*} S_{1}^{i}\left(S_{1}^{*} x\right)$ equals $S_{2}^{*} S_{1}^{*} x=S_{1}^{*} S_{2}^{*} x=0$ for $i=0$, and $S_{2}^{*} S_{1}^{i-1} x=0$ for $i \geq 1$. Obtain that $x \in S_{1} \bigcap_{i>0} \operatorname{ker} S_{2}^{*} S_{1}^{i}$. Then the maximal subspace of $\bigcap_{i \geq 0} \operatorname{ker} S_{2}^{*} S_{1}^{i}$ on which $\left.S_{1}\right|_{\bigcap_{i \geq 0}} \operatorname{ker} S_{2}^{*} S_{1}^{i}$ is unitary, namely $\bigcap_{m \geq 0} S_{1}^{m} \bigcap_{i \geq 0} \operatorname{ker} S_{2}^{*} S_{1}^{i}$ (by the theorem of Wold), coincides with $\bigcap_{m \geq 0} S_{1}^{m} \mathcal{H} \cap$ $\bigcap_{i>0} \operatorname{ker} S_{2}^{*} S_{1}^{i}$. The equivalence $(i) \Leftrightarrow(i i i)$ is proved.

The next step is to compute the maximal subspace reducing $S_{1}\left(I-S_{2} S_{2}^{*}\right)$ to a unitary operator (according to (4)). Proceed inductively by proving that $x \in \mathcal{H}$ satisfies

$$
\left\|\left[S_{1}\left(I-S_{2} S_{2}^{*}\right)\right]^{k} x\right\|=\|x\|=\left\|\left[S_{1}\left(I-S_{2} S_{2}^{*}\right)\right]^{* k} x\right\|, \quad 0 \leq k \leq n,
$$


if and only if $x \in \bigcap_{k=0}^{n-1} \operatorname{ker} S_{2}^{*} S_{1}^{k} \cap \bigcap_{k=0}^{n} S_{1}^{k} \mathcal{H}$. It is enough to apply successively the following classical result: if $P$ is an orthogonal projection on $\mathcal{H}$, then $x \in P \mathcal{H}$ if and only if $\|P x\|=\|x\|$. For $n=1,\left\|S_{1}\left(I-S_{2} S_{2}^{*}\right) x\right\|=\|x\|$ if and only if $x \in \operatorname{ker} S_{2}^{*}$ (take $\left.P=I-S_{2} S_{2}^{*}\right)$, while $\left\|\left(I-S_{2} S_{2}^{*}\right) S_{1}^{*} x\right\|=\|x\|$ if and only if $x \in \operatorname{ker}\left(S_{1} S_{2}\right)^{*} \cap S_{1} \mathcal{H}$ (take $P=S_{1}\left(I-S_{2} S_{2}^{*}\right) S_{1}^{*}$ and observe that $\left.P \mathcal{H}=S_{1} \operatorname{ker} S_{2}^{*}=\operatorname{ker}\left(S_{1} S_{2}\right)^{*} \cap S_{1} \mathcal{H}\right)$. Suppose now that the property is true for a given $n \geq 1$. Then (5) holds for $0 \leq k \leq n+1$ if and only if

$$
x \in \bigcap_{k=0}^{n-1} \operatorname{ker} S_{2}^{*} S_{1}^{k} \cap \bigcap_{k=0}^{n} S_{1}^{k} \mathcal{H},
$$

$$
\left\|S_{1}\left(I-S_{2} S_{2}^{*}\right)\left[S_{1}\left(I-S_{2} S_{2}^{*}\right)\right]^{n} x\right\|=\left\|\left[S_{1}\left(I-S_{2} S_{2}^{*}\right)\right]^{n} x\right\|
$$

(i.e., $\left.\left[S_{1}\left(I-S_{2} S_{2}^{*}\right)\right]^{n} x \in \operatorname{ker} S_{2}^{*}\right)$ and

$$
\left\|\left(I-S_{2} S_{2}^{*}\right) S_{1}^{*}\left[\left(I-S_{2} S_{2}^{*}\right) S_{1}^{*}\right]^{n} x\right\|=\left\|\left[\left(I-S_{2} S_{2}^{*}\right) S_{1}^{*}\right]^{n} x\right\|
$$

(i.e., $\left.\left[\left(I-S_{2} S_{2}^{*}\right) S_{1}^{*}\right]^{n} x \in \operatorname{ker}\left(S_{1} S_{2}\right)^{*} \cap S_{1} \mathcal{H}\right)$. The induction is completed by the remark that, for $x \in \bigcap_{k=0}^{n-1} \operatorname{ker} S_{2}^{*} S_{1}^{k} \cap \bigcap_{k=0}^{n} S_{1}^{k} \mathcal{H}$ we have $\left[S_{1}\left(I-S_{2} S_{2}^{*}\right)\right]^{n} x=S_{1}^{n} x$ (so $S_{1}^{n} x \in \operatorname{ker} S_{2}^{*}$, that is, $\left.x \in \operatorname{ker} S_{2}^{*} S_{1}^{n}\right)$ and $\left[\left(I-S_{2} S_{2}^{*}\right) S_{1}^{*}\right]^{n} x=S_{1}^{* n} x$ (so $S_{1}^{* n} x \in$ $\operatorname{ker}\left(S_{1} S_{2}\right)^{*} \cap S_{1} \mathcal{H}$, that is, $\left.x=S_{1}^{n} S_{1}^{* n} x=S_{1}^{n}\left(S_{1} S_{1}^{*} S_{1}^{* n} x\right)=S_{1}^{n+1} S_{1}^{* n+1} x \in S_{1}^{n+1} \mathcal{H}\right)$. Deduce that, by (4), the maximal subspace reducing $S_{1}\left(I-S_{2} S_{2}^{*}\right)$ to a unitary operator is $\bigcap_{m \geq 0} S_{1}^{m} \mathcal{H} \cap \bigcap_{i \geq 0} \operatorname{ker} S_{2}^{*} S_{1}^{i}$, the same as the maximal subspace reducing $\left.S_{1}\right|_{\cap_{i \geq 0}} \operatorname{ker} S_{2}^{*} S_{1}^{i}$ to a unitary operator. The equivalence $(i) \Leftrightarrow(i i)$ is proved.

Consequently:

Proposition 2.7. The following assertions are equivalent:

(i) $S$ is a weak bi-shift;

(ii) $S_{1}\left(I-S_{2} S_{2}^{*}\right), S_{2}\left(I-S_{1} S_{1}^{*}\right)$ and $S_{1} S_{2}$ are cnu;

$$
\bigcap_{m \geq 0} S_{1}^{m} \mathcal{H} \cap \bigcap_{i \geq 0} \operatorname{ker} S_{2}^{*} S_{1}^{i}=\bigcap_{n \geq 0} S_{2}^{n} \mathcal{H} \cap \bigcap_{j \geq 0} \operatorname{ker} S_{1}^{*} S_{2}^{j}=\bigcap_{n \geq 0}\left(S_{1} S_{2}\right)^{n} \mathcal{H}=\{0\} .
$$

The main result of this section is

Theorem 2.8. Let $V=\left(V_{1}, V_{2}\right)$ be a bi-isometry on $\mathcal{H}$. There is a unique orthogonal decomposition of the form

$$
\mathcal{H}=\mathcal{H}_{u u} \oplus \mathcal{H}_{u s} \oplus \mathcal{H}_{s u} \oplus \mathcal{H}_{w s}
$$

into reducing subspaces for $V$ such that $\left.V\right|_{\mathcal{H}_{u u}}$ is bi-unitary, $\left.V\right|_{\mathcal{H}_{u s}}$ is unitary-shift, $\left.V\right|_{\mathcal{H}_{s u}}$ is shift-unitary and $\left.V\right|_{\mathcal{H}_{w s}}$ is a weak bi-shift.

In addition, $\mathcal{H}_{u u}, \mathcal{H}_{u s}$ and $\mathcal{H}_{\text {su }}$ have the structure given by (11), (2) and respectively (3).

Proof. Define $\mathcal{H}_{u u}$ by (11), $\mathcal{H}_{u s}$ by (21) and $\mathcal{H}_{s u}$ by (3). If $\mathcal{H}=\mathcal{H}_{u}^{k} \oplus \mathcal{H}_{s}^{k}$ is the Wold decomposition associated to the isometry $V_{k}, k=1,2$, then $\mathcal{H}_{u u} \subset \mathcal{H}_{u}^{1} \cap \mathcal{H}_{u}^{2}$, $\mathcal{H}_{u s} \subset \mathcal{H}_{u}^{1} \cap \mathcal{H}_{s}^{2}$ and $\mathcal{H}_{s u} \subset \mathcal{H}_{s}^{1} \cap \mathcal{H}_{u}^{2}$. The orthogonal sum $\mathcal{H}_{u u} \oplus \mathcal{H}_{u s} \oplus \mathcal{H}_{s u}$ is then well-defined, and its orthogonal complement $\mathcal{H}_{w s}$ is also reducing for $V$. Since $\left.V\right|_{\mathcal{H}_{w s}}$ does not have reducing bi-unitary, unitary-shift or shift-unitary parts, we 
obtain

$$
\begin{gathered}
\left(\bigcap_{n \geq 0}\left(V_{1} V_{2}\right)^{n} \mathcal{H}\right) \cap \mathcal{H}_{w s}=\{0\}, \\
\left(\bigcap_{m \geq 0} V_{1}^{m}\left(\bigcap_{i \geq 0} \operatorname{ker} V_{2}^{*} V_{1}^{i}\right)\right) \cap \mathcal{H}_{w s}=\{0\}
\end{gathered}
$$

and

$$
\left(\bigcap_{n \geq 0} V_{2}^{n}\left(\bigcap_{j \geq 0} \operatorname{ker} V_{1}^{*} V_{2}^{j}\right)\right) \cap \mathcal{H}_{w s}=\{0\}
$$

According to Definition 2.5, $\left.V\right|_{\mathcal{H}_{w s}}$ is a weak bi-shift.

If $\mathcal{H}=\mathcal{H}_{u u}^{\prime} \oplus \mathcal{H}_{u s}^{\prime} \oplus \mathcal{H}_{s u}^{\prime} \oplus \mathcal{H}_{w s}^{\prime}$ is another decomposition into reducing summands such that $\left.V\right|_{\mathcal{H}_{u u}^{\prime}}$ is bi-unitary, $\left.V\right|_{\mathcal{H}_{u s}^{\prime}}$ is unitary-shift, $\left.V\right|_{\mathcal{H}_{s u}^{\prime}}$ is shift-unitary and $\left.V\right|_{\mathcal{H}_{w s}^{\prime}}$ is a weak bi-shift, then $\mathcal{H}_{u u}^{\prime} \subset \mathcal{H}_{u u}, \mathcal{H}_{u s}^{\prime} \subset \mathcal{H}_{u s}$ and $\mathcal{H}_{s u}^{\prime} \subset \mathcal{H}_{s u}$ by maximality (Propositions 2.1 2.3). Then $\mathcal{H}_{w s}^{\prime} \supset\left(\mathcal{H}_{u u} \ominus \mathcal{H}_{u u}^{\prime}\right) \oplus\left(\mathcal{H}_{u s} \ominus \mathcal{H}_{u s}^{\prime}\right) \oplus\left(\mathcal{H}_{s u} \ominus \mathcal{H}_{s u}^{\prime}\right)$. Since a weak bi-shift has null bi-unitary, unitary-shift and respectively shift-unitary maximal parts, we obtain $\mathcal{H}_{u u} \ominus \mathcal{H}_{u u}^{\prime}=\{0\}, \mathcal{H}_{u s} \ominus \mathcal{H}_{u s}^{\prime}=\{0\}$ and $\mathcal{H}_{s u} \ominus \mathcal{H}_{s u}^{\prime}=\{0\}$. The decomposition is unique.

There is a maximal bi-shift part in any bi-isometry (see [Su, [St], [GS1]). We give here a more precise structure:

Proposition 2.9. There exists a maximal subspace $\mathcal{H}_{s} \subset \mathcal{H}_{\text {ws }}$ of $\mathcal{H}$ reducing $V$ to a bi-shift. More precisely,

$$
\mathcal{H}_{s}=\bigoplus_{p \in \mathbb{Z}_{+}^{2}} V^{p}\left(\bigcap_{i \geq 0} \operatorname{ker} V_{2}^{*} V_{1}^{i} \cap \bigcap_{j \geq 0} \operatorname{ker} V_{1}^{*} V_{2}^{j}\right) .
$$

Proof. An easy computation shows that $\mathcal{W}=\bigcap_{i \geq 0} \operatorname{ker} V_{2}^{*} V_{1}^{i} \cap \bigcap_{j \geq 0} \operatorname{ker} V_{1}^{*} V_{2}^{j}$ is wandering for $V$. If we denote by $\mathcal{H}_{s}$ the orthogonal sum in (17), then $V_{k} \mathcal{H}_{s}=$ $\mathcal{H}_{s} \ominus\left(\bigoplus_{n \geq 0} V_{3-k}^{n} \mathcal{W}\right)$ and $V_{k}^{*} \mathcal{H}_{s}=\mathcal{H}_{s}, k=1,2$. Therefore, $\mathcal{H}_{s}$ reduces $V$ to a bi-shift having $\mathcal{W}$ as the defect subspace.

Suppose that $\mathcal{H}_{0}$ is a subspace of $\mathcal{H}$ reducing $V$ to a bi-shift. Since $\left.V\right|_{\mathcal{H}_{0}}$ is double commuting, we can use, as before, the geometrical structure in the Słociński decomposition:

$$
\mathcal{H}_{0}=\bigoplus_{p \in \mathbb{Z}_{+}^{2}} V^{p}\left(\operatorname{ker} V_{1}^{*} \cap \operatorname{ker} V_{2}^{*} \cap \mathcal{H}_{0}\right)
$$

Observe that $V_{2}^{*} V_{1}^{i} x=V_{1}^{i} V_{2}^{*} x=0(i \geq 0)$ and $V_{1}^{*} V_{2}^{j} x=V_{2}^{j} V_{1}^{*} x=0(j \geq 0)$, for any $x \in \operatorname{ker} V_{1}^{*} \cap \operatorname{ker} V_{2}^{*} \cap \mathcal{H}_{0}$. Then $\mathcal{H}_{0} \subset \mathcal{H}_{s}$, and the maximality is proved.

With the notation in the proof of Theorem 2.8 it is easy to observe that $\mathcal{H}_{s} \subset$ $\mathcal{H}_{s}^{1} \cap \mathcal{H}_{s}^{2}$. Consequently, $\mathcal{H}_{s} \perp \mathcal{H}_{u u} \oplus \mathcal{H}_{u s} \oplus \mathcal{H}_{s u}$ and, by Theorem 2.8, $\mathcal{H}_{s} \subset \mathcal{H}_{w s}$.

In any bi-isometry we can identify a double commuting part. More precisely:

Proposition 2.10. Let $V=\left(V_{1}, V_{2}\right)$ be a bi-isometry on $\mathcal{H}$. Then there exists a maximal subspace $\mathcal{H}_{d c}$ of $\mathcal{H}$ reducing $V$ to a double commuting bi-isometry. More precisely,

$$
\mathcal{H}_{d c}=\left\{x \in \mathcal{H} \mid V_{1}^{m} V_{1}^{* i} V_{2}^{n} V_{2}^{* j} x=V_{2}^{n} V_{2}^{* j} V_{1}^{m} V_{1}^{* i} x, m, n, i, j \geq 0\right\} .
$$


Proof. Define $\mathcal{H}_{d c}$ by (8). $\mathcal{H}_{d c}$ is a closed subspace, being an intersection of bounded operator kernels. For any $x \in \mathcal{H}_{d c}$ and $m, n, i, j \geq 0$, observe that

$$
V_{1}^{m} V_{1}^{* i} V_{2}^{n} V_{2}^{* j} V_{1} x=V_{1}^{m} V_{1}^{* i} V_{1} V_{2}^{n} V_{2}^{* j} x=V_{2}^{n} V_{2}^{* j} V_{1}^{m} V_{1}^{* i} V_{1} x
$$

and

$$
V_{1}^{m} V_{1}^{* i} V_{2}^{n} V_{2}^{* j} V_{1}^{*} x=V_{1}^{m} V_{1}^{* i} V_{1}^{*} V_{2}^{n} V_{2}^{* j} x=V_{2}^{n} V_{2}^{* j} V_{1}^{m} V_{1}^{* i} V_{1}^{*} x .
$$

Hence $\mathcal{H}_{d c}$ reduces $V_{1}$ and, similarly, also $V_{2}$. It is obvious that $\left.V\right|_{\mathcal{H}_{d c}}$ is double commuting and $\mathcal{H}_{d c}$ is maximal.

Corollary 2.11. The Stociński decomposition attached to $\left.V\right|_{\mathcal{H}_{d c}}$ is

$$
\mathcal{H}_{d c}=\mathcal{H}_{u u} \oplus \mathcal{H}_{u s} \oplus \mathcal{H}_{s u} \oplus \mathcal{H}_{s},
$$

the subspaces $\mathcal{H}_{u u}, \mathcal{H}_{u s}, \mathcal{H}_{s u}$ and $\mathcal{H}_{s}$ being given by (1)-(3) and (7).

We can now give another definition for the weak bi-shift:

Corollary 2.12. A bi-isometry $V$ on $\mathcal{H}$ is a weak bi-shift if and only if $\left.V\right|_{\mathcal{H}_{d c}}$ is a bi-shift.

\section{The ASSOCIATED DUAL BI-ISOMETRY}

Let $V=\left(V_{1}, V_{2}\right)$ be a bi-isometry on a Hilbert space $\mathcal{H}$. A pair $U=\left(U_{1}, U_{2}\right)$ of commuting unitary operators on a Hilbert space $\mathcal{K} \supset \mathcal{H}$ with $\left.U_{k}\right|_{\mathcal{H}}=V_{k}, k=1,2$ (extension), and $\mathcal{K}=\bigvee_{p \in \mathbb{Z}^{2}} U^{p} \mathcal{H}$ (minimality) is said to be the minimal unitary extension of $V$. Its existence is given by a result of Ito ([It), and the minimality condition assures its uniqueness up to an isomorphism.

Observe that $\tilde{\mathcal{H}}=\mathcal{K} \ominus \mathcal{H}$ is invariant under $U^{*}$.

Definition 3.1. The bi-isometry $\tilde{V}:=\left.U^{*}\right|_{\tilde{\mathcal{H}}}$ is said to be the dual bi-isometry associated to $V$ (see GS2]).

We can introduce some particular classes of bi-isometries by certain properties of their associated dual bi-isometries:

Definition 3.2. $V$ is said to be:

(1) dual double commuting if $\tilde{V}$ is double commuting (see [GG]);

(2) modified bi-shift if $V$ is completely non-unitary and $\tilde{V}$ is bi-shift (see [GS2]);

(3) modified weak bi-shift if $V$ is completely non-unitary and $\tilde{V}$ is weak bi-shift.

To prove some properties of the dual bi-isometry we need the following:

Lemma 3.3. (a) $V_{2}^{n} \operatorname{ker} V_{1}^{*} V_{2}^{n}=U_{1} \operatorname{ker} \tilde{V}_{1}^{*} \tilde{V}_{2}^{n}, n \geq 0$;

(b) $V_{2}^{n+1} \operatorname{ker} V_{1}^{*} V_{2}^{n}=V_{2}^{n+1} \mathcal{H} \cap \operatorname{ker}\left(V_{1} V_{2}\right)^{*}, n \geq 0$.

By symmetry, the same kind of results are also true if we switch the roles of $V_{1}$ and $V_{2}$.

Proof. (a) If $y \in \operatorname{ker} \tilde{V}_{1}^{*} \tilde{V}_{2}^{n}$, then $P_{\tilde{\mathcal{H}}} U_{1} U_{2}^{* n} y=\tilde{V}_{1}^{*} \tilde{V}_{2}^{n} y=0$; that is, $U_{1} U_{2}^{* n} y \in \mathcal{H}$. Moreover, $V_{1}^{*} V_{2}^{n} U_{1} U_{2}^{* n} y=V_{1}^{*} U_{1} y=P_{\mathcal{H}} y=0$.

Conversely, for $x \in \operatorname{ker} V_{1}^{*} V_{2}^{n}$, we obtain that $P_{\mathcal{H}} U_{1}^{*} U_{2}^{n} x=V_{1}^{*} V_{2}^{n} x=0$; that is, $U_{1}^{*} U_{2}^{n} x \in \tilde{\mathcal{H}}$. Moreover, $\tilde{V}_{1}^{*} \tilde{V}_{2}^{n} U_{1}^{*} U_{2}^{n} x=P_{\tilde{\mathcal{H}}} x=0$.

(b) Observe that, for $x \in V_{2}^{n+1} \mathcal{H}$ (or equivalently $x=V_{2}^{n+1} V_{2}^{* n+1} x$ ), $x \in$ $\operatorname{ker}\left(V_{1} V_{2}\right)^{*}$ if and only if $\left(V_{1} V_{2}\right)^{*} V_{2}^{n+1} V_{2}^{* n+1} x=0$, that is, $V_{2}^{* n+1} x \in \operatorname{ker} V_{1}^{*} V_{2}^{n}$.

Since ker $V_{2}^{*}$ is invariant under $V_{1}^{*}$, we can consider $\left.V_{1}^{*}\right|_{\text {ker } V_{2}^{*}}$ as a contraction on $\operatorname{ker} V_{2}^{*}$ and, similarly, $\left.V_{2}^{*}\right|_{\operatorname{ker} V_{1}^{*}}$ on $\operatorname{ker} V_{1}^{*}$. 
Proposition 3.4. (i) $\tilde{V}$ is always completely non-unitary (that is, $\tilde{V}_{1} \tilde{V}_{2}$ is a shift).

(ii) $V$ is completely non-unitary if and only if $\tilde{\tilde{V}}=V$.

(iii) The following assertions are equivalent:

(a) $V$ is dual double commuting;

(b) $\operatorname{ker} V_{1}^{*} \perp \operatorname{ker} V_{2}^{*}$;

(c) $V_{1} \operatorname{ker} V_{2}^{*} \supset \operatorname{ker} V_{2}^{*}\left(\right.$ or $\left.V_{2} \operatorname{ker} V_{1}^{*} \supset \operatorname{ker} V_{1}^{*}\right)$;

(d) $\left.V_{1}^{*}\right|_{\operatorname{ker} V_{2}^{*}}\left(\right.$ or $\left.V_{2}^{*}\right|_{\operatorname{ker} V_{1}^{*}}$ ) is isometric.

Proof. (i) and (ii) have been proved in [Po Remark 3.5].

(iii) By Lemma 3.3( $(a)$ for $n=0, \operatorname{ker} V_{1}^{*} \perp \operatorname{ker} V_{2}^{*}$ if and only if $\tilde{V}_{2} \operatorname{ker} \tilde{V}_{1}^{*} \perp$ $\tilde{V}_{1}$ ker $\tilde{V}_{2}{ }^{*}$ or, equivalently, $\left[\tilde{V}_{1}\left(I-\tilde{V}_{2} \tilde{V}_{2}{ }^{*}\right)\right]^{*}\left[\tilde{V}_{2}\left(I-\tilde{V}_{1} \tilde{V}_{1}{ }^{*}\right)\right]=0$ (in operator form). By an easy computation we obtain $\tilde{V}_{1}^{*} \tilde{V}_{2}=\tilde{V}_{2} \tilde{V}_{1}^{*}$. For the second part, by Lemma 3.3 (b), $V_{1} \operatorname{ker} V_{2}^{*} \supset \operatorname{ker} V_{2}^{*}$ if and only if $V_{1} \mathcal{H} \supset \operatorname{ker} V_{2}^{*}$ or, equivalently, $\operatorname{ker} V_{1}^{*} \perp$ $\operatorname{ker} V_{2}^{*}$. Finally, $\left.V_{1}^{*}\right|_{\operatorname{ker} V_{2}^{*}}$ is isometric if and only if

$$
0=\left\|\left(I-V_{2} V_{2}^{*}\right) x\right\|^{2}-\left\|V_{1}^{*}\left(I-V_{2} V_{2}^{*}\right) x\right\|^{2}=\left\|\left(I-V_{1} V_{1}^{*}\right)\left(I-V_{2} V_{2}^{*}\right) x\right\|^{2}, \quad x \in \mathcal{H},
$$

that is, if and only if $\operatorname{ker} V_{1}^{*} \perp \operatorname{ker} V_{2}^{*}$.

Using Proposition 3.4 $(i)$, write the Wold-type decomposition in Theorem 2.8 for $\tilde{V}$ instead of $V$ :

$$
\tilde{\mathcal{H}}=\tilde{\mathcal{H}}_{u s} \oplus \tilde{\mathcal{H}}_{s u} \oplus \tilde{\mathcal{H}}_{w s} .
$$

Then $\tilde{\mathcal{H}}_{u s}=\bigoplus_{n \geq 0} \tilde{V}_{2}^{n} \tilde{\mathcal{W}}_{2}=\bigoplus_{n \leq 0} U_{2}^{n} \tilde{\mathcal{W}}_{2}$ with $\tilde{\mathcal{W}}_{2}=\bigcap_{m \geq 0} \tilde{V}_{1}^{m}\left(\bigcap_{i \geq 0} \operatorname{ker} \tilde{V}_{2}^{*} \tilde{V}_{1}^{i}\right)$ and, by passing to the dual, $\widetilde{\tilde{\mathcal{H}}_{u s}}=\bigoplus_{n \in \mathbb{Z}} U_{2}^{n} \tilde{\mathcal{W}}_{2} \ominus \tilde{\mathcal{H}}_{u s}=\bigoplus_{n \geq 0} U_{2}^{n}\left(U_{2} 2 \tilde{\mathcal{W}}_{2}\right)$. Observe that $U_{1}^{*} \bigcap_{i \geq 0} \operatorname{ker} V_{2}^{*} V_{1}^{i} \supset \bigcap_{i \geq 0} \operatorname{ker} V_{2}^{*} V_{1}^{i}$ and, by duality, $U_{1} \bigcap_{i \geq 0} \operatorname{ker} \tilde{V}_{2}^{*} \tilde{V}_{1}^{i} \supset$ $\bigcap_{i \geq 0} \operatorname{ker} \tilde{V}_{2}{ }^{*} \tilde{V}_{1}{ }^{i}$. Use this observation and Lemma3.3(a) (more precisely, ker $\tilde{V}_{2}{ }^{*} \tilde{V}_{1}{ }^{i}$ $\left.=\bar{U}_{2}^{*} U_{1}^{i} \operatorname{ker} V_{2}^{*} V_{1}^{i}\right)$ to prove that

$$
\begin{aligned}
& U_{2} \tilde{\mathcal{W}}_{2}=U_{2} \bigcap_{m \in \mathbb{Z}} U_{1}^{m}\left(\bigcap_{i \geq 0} \operatorname{ker} \tilde{V}_{2}{ }^{*} \tilde{V}_{1}^{i}\right) \\
& \stackrel{m+i=p}{=} \bigcap_{p \in \mathbb{Z}, i \geq 0} U_{1}^{p} \operatorname{ker} V_{2}^{*} V_{1}^{i} \\
&=\bigcap_{p \geq 0} V_{1}^{p} \bigcap_{i \geq 0} \operatorname{ker} V_{2}^{*} V_{1}^{i} .
\end{aligned}
$$

Deduce $\widetilde{\tilde{\mathcal{H}}_{u s}}=\mathcal{H}_{u s}$ and, by symmetry, $\widetilde{\tilde{\mathcal{H}}_{s u}}=\mathcal{H}_{s u}$. Therefore, by duality, relation (9) becomes

$$
\mathcal{H}=\mathcal{H}_{u u} \oplus \mathcal{H}_{u s} \oplus \mathcal{H}_{s u} \oplus \widetilde{\tilde{\mathcal{H}}_{w s}}
$$

that is, $\widetilde{\tilde{\mathcal{H}}_{w s}}=\mathcal{H}_{w s}$. In other words:

Corollary 3.5. $V$ is a weak bi-shift if and only if $V$ is a modified weak bi-shift.

As observed earlier (Proposition 2.9 cf. [Su], [S1], GS1]), the weak bi-shift part contains a maximal bi-shift. Then $\mathcal{H}_{m}:=\widetilde{\tilde{\mathcal{H}}}_{s}$ is the maximal modified bi-shift subspace and is contained in $\widetilde{\tilde{\mathcal{H}}_{w s}}=\mathcal{H}_{w s} . \mathcal{H}_{m}$ was already considered in GS2. We give here a more precise structure: 
Corollary 3.6. There exists a maximal subspace $\mathcal{H}_{m} \subset \mathcal{H}_{\text {ws }}$ of $\mathcal{H}$ reducing $V$ to a modified bi-shift. More precisely,

$$
\mathcal{H}_{m}=\bigoplus_{p \in \mathbb{Z}^{2} \backslash(-\infty,-1]^{2}} V^{p}\left(\bigcap_{m \geq 0} V_{1}^{m} \mathcal{H} \cap \bigcap_{n \geq 0} V_{2}^{n} \mathcal{H} \cap \operatorname{ker}\left(V_{1} V_{2}\right)^{*}\right)
$$

(according to the notation for $V^{p}$ in Section 1$)$.

Proof. $\tilde{\mathcal{H}}_{s}=\bigoplus_{p \in \mathbb{Z}_{+}^{2}} \tilde{V}^{p}\left(\bigcap_{i \geq 0} \operatorname{ker} \tilde{V}_{2}^{*} \tilde{V}_{1}^{i} \cap \bigcap_{j \geq 0} \operatorname{ker} \tilde{V}_{1}^{*} \tilde{V}_{2}^{j}\right)$ by Proposition[2.9 Then $\mathcal{H}_{m}:=\widetilde{\tilde{\mathcal{H}}}_{s}=\bigoplus_{p \in \mathbb{Z}^{2} \backslash\left\{-\mathbb{Z}_{+}^{2}\right\}} U^{p}\left(\bigcap_{i \geq 0} \operatorname{ker} \tilde{V}_{2}^{*} \tilde{V}_{1}^{i} \cap \bigcap_{j \geq 0} \operatorname{ker} \tilde{V}_{1}^{*} \tilde{V}_{2}^{j}\right)$. Use again Lemma $3.3(a)$ and $(b)$ to see that

$$
\begin{aligned}
\mathcal{H}_{m} & =\bigoplus_{p \in \mathbb{Z}^{2} \backslash\left\{-\mathbb{Z}_{+}^{2}\right\}} U^{p-(1,1)}\left(\bigcap_{i \geq 0} V_{1}^{i+1} \operatorname{ker} V_{2}^{*} V_{1}^{i} \cap \bigcap_{j \geq 0} V_{2}^{j+1} \operatorname{ker} V_{1}^{*} V_{2}^{j}\right) \\
& =\bigoplus_{p \in \mathbb{Z}^{2} \backslash\left\{-\mathbb{Z}_{+}^{2}\right\}} U^{p-(1,1)}\left(\bigcap_{m \geq 0} V_{1}^{m} \mathcal{H} \cap \bigcap_{n \geq 0} V_{2}^{n} \mathcal{H} \cap \operatorname{ker}\left(V_{1} V_{2}\right)^{*}\right) .
\end{aligned}
$$

Moreover, $\mathcal{H}_{m} \subset \mathcal{H}$ implies $U^{p-(1,1)} x=P_{\mathcal{H}} U^{p-(1,1)} x=V^{p-(1,1)} x$, for any $x \in$ $\bigcap_{m \geq 0} V_{1}^{m} \mathcal{H} \cap \bigcap_{n \geq 0} V_{2}^{n} \mathcal{H} \cap \operatorname{ker}\left(V_{1} V_{2}\right)^{*}$ and $p \notin(-\infty, 0]^{2}$. (111) is proved.

Remark 3.7. (a) Since $\widetilde{\tilde{\mathcal{H}}_{m}}=\mathcal{H}_{s}$, the method above would not provide another reducing subspace of $\mathcal{H}_{w s}$.

(b) If $V_{1}$ (resp. $\left.V_{2}\right)$ is a shift, then $\mathcal{H}_{u u}=\mathcal{H}_{u s}\left(\right.$ resp. $\left.\mathcal{H}_{s u}\right)=\mathcal{H}_{m}=\{0\}$; if $V_{1}$ (resp. $V_{2}$ ) is unitary, then $\mathcal{H}_{s u}$ (resp. $\left.\mathcal{H}_{u s}\right)=\mathcal{H}_{w s}=\{0\}$.

(c) If $V$ is dual double commuting ( $\operatorname{ker} V_{1}^{*} \perp \operatorname{ker} V_{2}^{*}$ according to Proposition 3.4), then the decomposition (10) becomes the one given in GG]:

$$
\mathcal{H}=\mathcal{H}_{u u} \oplus \mathcal{H}_{u s} \oplus \mathcal{H}_{s u} \oplus \mathcal{H}_{m}
$$

We can give here a more precise structure than in $\mathrm{Po}$. Observe that, in this case, by Lemma 3.3( $(a)$ applied for $n=0, U_{2} \bigcap_{m \geq 0} \tilde{V}_{1}^{m} \operatorname{ker} \tilde{V}_{2}^{*}=\bigcap_{m \leq 0} U_{1}^{m} \operatorname{ker} V_{2}^{*}=$ $\bigcap_{m \geq 0} \operatorname{ker} V_{2}^{*} V_{1}^{m}$, and this implies that

$$
\mathcal{H}_{u s}=\bigoplus_{n \geq 0} V_{2}^{n}\left(\bigcap_{i \geq 0} \operatorname{ker} V_{2}^{*} V_{1}^{i}\right) .
$$

Similarly,

$$
\mathcal{H}_{s u}=\bigoplus_{m \geq 0} V_{1}^{m}\left(\bigcap_{j \geq 0} \operatorname{ker} V_{1}^{*} V_{2}^{j}\right) .
$$

Moreover, $\operatorname{ker} V_{2}^{*} V_{1}^{i}=V_{1} \operatorname{ker} V_{2}^{*} V_{1}^{i+1}(i \geq 0)$ since, on the one side, $x \in V_{1} \operatorname{ker} V_{2}^{*} V_{1}^{i+1}$ $\left(x=V_{1} V_{1}^{*} x\right.$ and $\left.V_{2}^{*} V_{1}^{i+1} V_{1}^{*} x=0\right)$ implies $x \in \operatorname{ker} V_{2}^{*} V_{1}^{i}$, and, on the other side, $x \in \operatorname{ker} V_{2}^{*} V_{1}^{i}$ implies $x \in V_{1}^{* i} \operatorname{ker} V_{2}^{*} \subset V_{1}^{* i} V_{1}^{i+1} \operatorname{ker} V_{2}^{*}=V_{1} \operatorname{ker} V_{2}^{*}$ (since by Proposition $\left.3.4 \operatorname{ker} V_{2}^{*} \subset V_{1} \operatorname{ker} V_{2}^{*}\right)$, that is, $x=V_{1} V_{1}^{*} x \in V_{1} \operatorname{ker} V_{2}^{*} V_{1}^{i+1}$. Then $V_{1}^{i+1} \operatorname{ker} V_{2}^{*} V_{1}^{i}=V_{1} \operatorname{ker} V_{2}^{*}(i \geq 0), V_{2}^{j+1} \operatorname{ker} V_{1}^{*} V_{2}^{j}=V_{2} \operatorname{ker} V_{1}^{*}(j \geq 0)$, and so, by Corollary 3.6.

$$
\mathcal{H}_{m}=\bigoplus_{p \in \mathbb{Z}^{2} \backslash(-\infty,-1]^{2}} V^{p}\left(V_{1} \operatorname{ker} V_{2}^{*} \cap V_{2} \operatorname{ker} V_{1}^{*}\right)
$$

(d) If $V$ is simultaneously double and dual double commuting $\left(V_{1} \operatorname{ker} V_{2}^{*}=\operatorname{ker} V_{2}^{*}\right.$ or, equivalently, $\left.V_{2} \operatorname{ker} V_{1}^{*}=\operatorname{ker} V_{1}^{*}\right)$, then, using again the notation in the proof of 
Theorem 2.8, $\mathcal{H}_{u s}=\mathcal{H}_{s}^{2}, \mathcal{H}_{s u}=\mathcal{H}_{s}^{1}$ and $\mathcal{H}_{w s}=\{0\}$. More precisely, the decomposition (6) becomes

$$
\mathcal{H}=\left(\mathcal{H}_{u}^{1} \cap \mathcal{H}_{u}^{2}\right) \oplus \mathcal{H}_{s}^{2} \oplus \mathcal{H}_{s}^{1} \oplus\{0\} .
$$

Modified bi-shifts can be characterized as follows:

Proposition 3.8. The following assertions are equivalent:

(i) $V$ is a modified bi-shift.

(ii) $V$ is dual double commuting and

$$
\mathcal{H}=\bigoplus_{p \in \mathbb{Z}^{2} \backslash(-\infty,-1]^{2}} V^{p}\left(V_{1} \operatorname{ker} V_{2}^{*} \cap V_{2} \operatorname{ker} V_{1}^{*}\right) .
$$

(iii) $\left.\left.V_{1}^{*}\right|_{\operatorname{ker} V_{2}^{*}, V_{2}^{*}}\right|_{\operatorname{ker} V_{1}^{*}}$ and $V_{1} V_{2}$ are shifts.

Proof. ( $i)$ and (ii) are equivalent by Remark 3.7(c). In addition, by Remark 2.4, if $V$ is completely non-unitary, then $V$ is a modified bi-shift if and only if $\left.\tilde{V}_{1}\right|_{\operatorname{ker} \tilde{V}_{2}^{*}},\left.\tilde{V}_{2}\right|_{\text {ker } \tilde{V}_{1}^{*}}$ and $\tilde{V}_{1} \tilde{V}_{2}$ are shifts. The proof is completed by the observation that $\left.\tilde{V}_{1}\right|_{\operatorname{ker} \tilde{V}_{2}^{*}}\left(\right.$ respectively $\left.\left.\tilde{V}_{2}\right|_{\operatorname{ker} \tilde{V}_{1}^{*}}\right)$ is a shift if and only if $\left.V_{1}^{*}\right|_{\operatorname{ker} V_{2}^{*}}$ (respectively $\left.\left.V_{2}^{*}\right|_{\operatorname{ker} V_{1}^{*}}\right)$ is a shift. We just have to see that, by Lemma 3.3 $(a)$, the relation

$$
\operatorname{ker} \tilde{V}_{2}^{*}=\bigoplus_{m \geq 0} \tilde{V}_{1}^{m}\left(\operatorname{ker} \tilde{V}_{2}^{*} \ominus \tilde{V}_{1}^{*} \operatorname{ker} \tilde{V}_{2}^{*}\right)
$$

can be rewritten as

$$
\operatorname{ker} V_{2}^{*}=\bigoplus_{m \geq 0} U_{1}^{* m}\left(\operatorname{ker} V_{2}^{*} \ominus U_{1}^{*} \operatorname{ker} V_{2}^{*}\right) .
$$

Moreover, $U_{1}^{*} \operatorname{ker} V_{2}^{*} \subset \mathcal{H}$ (and consequently $U_{1}^{*} \operatorname{ker} V_{2}^{*}=V_{1}^{*} \operatorname{ker} V_{2}^{*}$ ) if $V$ is dual double commuting.

Example 3.9. We shall consider the example given in [S1]. Let $\mathcal{H}$ be a Hilbert space having an orthonormal basis of the form $\left\{e_{i, j} \mid i \in \mathbb{Z}_{+}\right.$or $\left.j \in \mathbb{Z}_{+}\right\}$. The pair $V=\left(V_{1}, V_{2}\right)$ defined on $\mathcal{H}$ by

$$
V_{1} e_{i, j}=e_{i+1, j} \text { and } V_{2} e_{i, j}=e_{i, j+1}(i \geq 0 \text { or } j \geq 0)
$$

is clearly a cnu bi-isometry. It is easy to see that

$$
\operatorname{ker} V_{1}^{*}=\bigvee\left\{e_{0, j} \mid j \leq-1\right\} \perp \bigvee\left\{e_{i, 0} \mid i \leq-1\right\}=\operatorname{ker} V_{2}^{*},
$$

that is, $\left.V_{1}^{*}\right|_{\operatorname{ker} V_{2}^{*}}$ and $\left.V_{2}^{*}\right|_{\operatorname{ker} V_{1}^{*}}$ are isometries. They are shifts, since $V_{1}^{* m} \operatorname{ker} V_{2}^{*}=$ $\bigvee\left\{e_{i, 0} \mid i \leq-m-1\right\}$ implies

$$
\bigcap_{m \geq 0} V_{1}^{* m} \operatorname{ker} V_{2}^{*}=\{0\}
$$

and, similarly,

$$
\bigcap_{n \geq 0} V_{2}^{* n} \operatorname{ker} V_{1}^{*}=\{0\}
$$

It follows by Proposition 3.8 that $V$ is a modified bi-shift.

As proved in [GS1] (or deduced directly following (77) and (11)), $\mathcal{H}_{s} \perp \mathcal{H}_{m}$. Then, by Propositions [2.1 2.3, 2.9, Theorem [2.8 and Corollary [3.6, we can conclude the following theorem. 
Theorem 3.10. Let $V$ be a bi-isometry on $\mathcal{H}$. There is a unique orthogonal decomposition of the form

$$
\mathcal{H}=\mathcal{H}_{u u} \oplus \mathcal{H}_{u s} \oplus \mathcal{H}_{s u} \oplus \mathcal{H}_{s} \oplus \mathcal{H}_{m} \oplus \mathcal{H}_{e}
$$

into subspaces of $\mathcal{H}$ reducing $V$ and maximal on which $V$ is, respectively, bi-unitary, unitary-shift, shift-unitary, bi-shift or modified bi-shift.

Since $\mathcal{H}_{e}$ vanishes in some important cases (for example, if $V$ is double or dual double commuting), we call $\left.V\right|_{\mathcal{H}_{e}}$ the evanescent part of $V$.

We give some necessary and sufficient conditions on $V$ in order to have no evanescent part:

Proposition 3.11. Let $V$ be a bi-isometry on $\mathcal{H}$. Then

$$
\mathcal{H}=\mathcal{H}_{\text {uu }} \oplus \mathcal{H}_{\text {us }} \oplus \mathcal{H}_{\text {su }} \oplus \mathcal{H}_{s} \oplus \mathcal{H}_{m}
$$

if and only if

$$
\begin{gathered}
\left(I-V_{1} V_{1}^{*}\right)\left(I-V_{2} V_{2}^{*}\right) \bigcap_{m \geq 0} V_{1}^{m} \mathcal{H}=\{0\}, \\
V_{2}^{*} V_{1}^{i}\left(I-V_{1} V_{1}^{*}\right) V_{1}^{* n}\left(I-V_{2} V_{2}^{*}\right)\left(I-V_{1} V_{1}^{*}\right)=0,
\end{gathered}
$$

and

$$
V_{1}^{*} V_{2}^{j}\left(I-V_{1} V_{1}^{*}\right) V_{1}^{* n}\left(I-V_{2} V_{2}^{*}\right)\left(I-V_{1} V_{1}^{*}\right)=0,
$$

for all $n, i, j \geq 0$.

Proof. Just observe that there exists an orthogonal decomposition of the form (12) if and only if $\left.V\right|_{\mathcal{H} \ominus \mathcal{H}_{s}}$ is dual double commuting, that is,

$$
\operatorname{ker} V_{2}^{*} \cap\left(\mathcal{H} \ominus \mathcal{H}_{s}\right) \subset V_{1}\left(\mathcal{H} \ominus \mathcal{H}_{s}\right)
$$

by Proposition 3.4 (iii). Equivalently, by passing to orthogonal complements,

$$
\operatorname{ker} V_{1}^{*} \subset V_{2} \mathcal{H} \oplus \bigoplus_{m \geq 0} V_{1}^{m} \mathcal{W} \text {, with } \mathcal{W}=\bigcap_{i \geq 0} \operatorname{ker} V_{2}^{*} V_{1}^{i} \cap \bigcap_{j \geq 0} \operatorname{ker} V_{1}^{*} V_{2}^{j} .
$$

(13) can be rewritten as $\left(I-V_{2} V_{2}^{*}\right)\left(I-V_{1} V_{1}^{*}\right) \mathcal{H} \subset \bigoplus_{m>0} V_{1}^{m} \mathcal{W}$ or, equivalently, $\left(I-V_{1} V_{1}^{*}\right)\left(I-V_{2} V_{2}^{*}\right) \bigcap_{m \geq 0} V_{1}^{m} \mathcal{H}=\{0\}$ and $\left(I-V_{1} V_{1}^{*}\right) V_{1}^{* n}\left(I-V_{2} V_{2}^{*}\right)\left(I-V_{1} V_{1}^{*}\right) \mathcal{H} \subset$ $\mathcal{W}(n \geq 0)$. The conclusion follows by the definition of $\mathcal{W}$.

In some other cases $V$ can have only evanescent part:

Example 3.12. Let $S$ be a shift on $\mathcal{H}$ and $V=(S, S)$. Then $V$ has no double commuting part. Moreover, by Remark [3.7 (b), the modified bi-shift part is also null. Then $\mathcal{H}=\mathcal{H}_{e}$, as required.

\section{ACKNOWLEDGMENTS}

The author wants to express his gratitude to Professor Bernard Chevreau and Wing Suet Li for useful suggestions and comments. The author is indebted to the referee for calling attention to some misprints and to some too concise proofs. 


\section{REFERENCES}

[BCL] C. A. Berger, L. A. Coburn and A. Lebow, Representation and Index Theory for $\mathbb{C}^{*}$ Algebras Generated by Commuting Isometries, J. Funct. Anal. 27 (1978), 51-99. MR 57:7251

[GG] D. Gaşpar and P. Gaşpar, Wold decompositions and the unitary model for bi-isometries, to appear.

[GS1] D. Gaspar and N. Suciu, Intertwining properties of isometric semigroups and Wold type decompositions, Operator Theory: Adv. and Appl. 24, Birkhäuser, Basel, 1987, pp. 183193. MR 89a:47066

[GS2] D. Gaşpar and N. Suciu, On the Wold decomposition of isometric semigroups, in Approximation Theory and Functional Analysis, Internat. Schriftenreihe Numer. Math. 65, Birkhäuser Verlag, Basel, 1984, pp. 99-108. MR 87g:47080

[It] T. Ito, On the commutative family of subnormal operators, J. Fac. Sci. Hokkaido Univ. Ser. I 14 (1958), 1-15. MR 21:5902

[Ko] M. Kosiek, Functional calculus and common invariant subspaces, Uniwersytet Jagiellonski Wydanie I, Krakow, 2001.

[La] H. Langer, Ein Zerspaltungssatz für Operatoren in Hilbertraum, Acta Math. Acad. Sci. Hungar. 12 (1961), 441-445. MR 25:3378

[NF1] B. Sz.-Nagy and C. Foias, Harmonic Analysis of Operators in Hilbert Space, NorthHolland, Amsterdam, 1970. MR 43:947

[NF2] B. Sz.-Nagy and C. Foiaş, Sur les contractions de l'espace de Hilbert. IV, Acta Sci. Math. (Szeged) 21 (1960), 251-259. MR 23:A3445

[Po] D. Popovici, On the structure of c.n.u. bi-isometries. II, Acta Sci. Math. (Szeged) 68 (2002), 329-347. MR 2003f:47018

[St] M. Słociński, On the Wold-type decomposition of a pair of commuting isometries, Ann. Polon. Math. 37 (1980), 255-262. MR 83e:47031

[Su] I. Suciu, On the semi-groups of isometries, Studia Math. 30 (1968), 101-110. MR 37:4671

[Wo] H. Wold, A study in the analysis of stationary time series, Almqvist and Wiksell, Stockholm, 1938 (2nd ed., 1954). MR 15:811d

Department of Mathematics, University of the West Timişoara, RO-300223 Timişoara, BD. Vasile PÂrvan NR. 4, Romania

E-mail address: popovici@math.uvt.ro 